\title{
7 Zusammenfassung
}

Die städtebaulichen Prozesse der 1950er- bis 60er-Jahre in Samarkand haben maßgeblich zu seiner Gestalt und späteren Entwicklung beigetragen. Dabei waren sie weniger ein Ausdruck einer einheitlichen Planung, sondern vielmehr der komplexen Gemengelage bei der diverse, einander widersprechende Aufgaben, Interessen und Bedürfnisse zwischen unterschiedlichen Akteuren verhandelt wurden.

Der sowjetische Staat war insbesondere nach dem Zweiten Weltkrieg mit einer massiven Wohnungsnot konfrontiert, wodurch die Führung zu Kompromissen und pragmatischen Lösungen gezwungen war. Sowohl die Wiederaufbaumaßnahmen nach dem Krieg als auch das Massenwohnungsbauprogramm von Khrushchev nach 1957 ließen in großem Maße Eigeninitiative von Bürgern zu. Zu einem wenig bekannten Bestandteil des unionsweiten Wohnungsbauprogramms gehörte die Unterstützung der Bürger beim Bau von privaten Häusern mit Landund Kreditzuteilungen. Darüber hinaus wurden eigenmächtig errichtete Häuser von den Behörden weitgehend toleriert. Während die Umsetzung der offiziellen Maßnahmen von Republik zu Republik variierte, stieß die Förderung des privaten Hausbaus in der Usbekischen SSR auf besonders viel Zuspruch - die Republik hatte den höchsten Anteil an privaten Wohnhäusern im Unionsvergleich und behielt die Position über Jahrzehnte hinweg, auch lange nachdem die Förderung 1962 nach und nach eingestellt wurde. Samarkand verkörpert das Ergebnis dieser Politik wie keine andere Stadt.

In dieser Arbeit untersuchte ich die Mechanismen hinter der Entstehung dieser Struktur, die formellen wie informellen Formen der individuellen Baupraxis, ihre Bedeutung bei der Bewältigung der Wohnungskrise sowie die damit verbundenen Konflikte und ihre weitreichenden Folgen für die Struktur der Stadt.

Die sowjetische Führung unter Khrushchev verfolgte hochgesteckte Ziele für die Entwicklung der Städte in der gesamten Sowjetunion - Wohnungsbau, Modernisierung und Industrialisierung sollten die Lebensqualität ihrer Einwohner radikal verbessern. In einer Stadt wie Samarkand mit bereits vorhandener historischen Stadtstruktur, knappem Bauland und zudem einer Infrastruktur und Bauindustrie, die erst aufgebaut werden mussten - gerieten diese Ziele und vor allem Maßnahmen zu ihrer Erfüllung häufig in Konflikt miteinander. Die Ursachen dafür lagen sowohl in unterschiedlichen Programmatiken (Etagenhäuser versus Eigenheime) als auch in der allgemeinen Ressourcenknappheit - der Finanzen, der Arbeitskräfte, des Baulandes.

2 Open Access. (C) 2021 Marya Petrova, publiziert von De Gruyter. (c))BY-NC-ND Dieses Werk ist lizenziert unter der Creative Commons Attribution-NonCommercial-NoDerivatives 4.0 International Lizenz.

https://doi.org/10.1515/9783110669367-007 
Die großzügige Parzellenzuteilung an Privatpersonen zwischen 1957 und 1962 zusammen mit einer vergleichbaren Zahl von eigenmächtig errichteten Häusern leistete kurzfristig gewisse Abhilfe in der Bewältigung der Wohnungsnot. Lange Zeit mangelte es an Alternativen - die Kapazitäten für den industriellen Wohnungsbau sollten erst geschaffen werden, bzw. die ersten Wohnungen nahmen wenig Rücksicht auf die lokalen klimatischen oder demografischen Anforderungen. Der Staat billigte seinen Bürgern Freiraum und eine gewisse Autonomie zu (,permitted autonomy“, Smith) und förderte diese sogar, da sie bei der Bekämpfung der Wohnungsnot half. Doch diese Art Kooperation war im Fall von Samarkand nur schwer zu kontrollieren - die Nachfrage seitens der Bürger überstieg bald die vorhandenen Kapazitäten an Bauland, gleichzeitig stieg die Zahl der eigenmächtig gebauten Häuser.

Die Stadtverwaltung stand vor einem schwer lösbaren Problem: Die knappen Flächen sollten auch für Infrastruktur, Industrie und den Massenwohnungsbau zur Verfügung gestellt werden. Ohne eigenes Bauland war die Stadt jedoch auf die Kolchosen angewiesen, die der Abtrennung ihrer Territorien zustimmen mussten, wogegen sie sich aber zu wehren versuchten. Die umstrittenen Besitzverhältnisse, gesetzliche Rückendeckung seitens des Landwirtschaftsministeriums sowie die gewichtige Position einiger Vorsitzender versah sie mit dem „längeren Hebel“ bei der Frage der Verteilung von wertvollen Territorien. Das Land wurde zur knappen „Ware“, das die Kolchosen eigenmächtig auch an Privatpersonen verteilten, und das dadurch den städtischen Bauvorhaben fehlte.

Die umfassenden Zuständigkeiten der Stadtverwaltung standen im Kontrast zu einer schwachen Position gegenüber den Ministerien, die ihre eigenen Interessen durchzusetzen versuchten. Zugleich unterlagen die Akteure in der Verwaltung selbst unterschiedlichen Zwängen, bei denen sie zwischen Einflussnahme und Interessenskonflikten manövrieren mussten. Die Stadtentwicklung selbst sollte formal durch einen Generalplan reguliert werden, doch die beschriebenen Konflikte einerseits, aber auch die starre Konzeption und Rücksichtlosigkeit gegenüber dem existierenden Kontext und den Bedürfnissen der Einwohner machte ihn schnell zur Makulatur. Das Ergebnis waren Kompromisse und kurzfristige Provisorien, aus denen schnell schwer zu beseitigende Tatsachen wurden.

Das Ergebnis dieser Arbeit soll keine bloße Nacherzählung eines Mangelnarrativs über die Defizite des sowjetischen Systems sein. Wichtiger für das Verständnis der Entwicklung von Samarkand ist eine Perspektive auf die Komplexität der Beziehungen und Aushandlungsprozesse und damit ein Blick auf weniger prominente Akteure. Die existierenden Rahmenbedingungen haben individuelles Handeln auf unterschiedlichen Ebenen ermöglicht - viele Bürger haben ihre (wenn 
auch nur erlaubte) Autonomie zu nutzen gewusst: Sie setzten ihre Interessen durch, lösten ihre Wohnungsnot weitgehend selbstständig und verschoben damit auch den Geltungsbereich der staatlichen (Bau)Pläne.

Samarkand zeichnete sich durch eine besondere Widerstandsfähigkeit gegenüber den sowjetischen Konzeptionen von einer modernen Großstadt aus. Die Wünsche und Bedürfnisse eines erheblichen Teils seiner Bewohner nach einem eigenen Haus mit Garten halfen eine Stadtstruktur zu erhalten, die von den offiziellen Stadtplanern als rückständig gebrandmarkt wurde, die aber unter den Gegebenheiten des sowjetischen Systems allen modernistischen Vorstellungen zum Trotz erstaunlich lebendig blieb. 